\title{
PERFORMANCE OF DUAL-HOP RELAYING OVER SHADOWED RICEAN FADING CHANNELS
}

\author{
Aleksandra M. CVETKOVIĆ - Jelena ANASTASOV \\ Stefan PANIĆ — Mihajlo STEFANOVIĆ — Dejan MILIĆ *
}

\begin{abstract}
In this paper, an analytical approach for evaluating performance of dual-hop cooperative link over shadowed Ricean fading channels is presented. New lower bound expressions for the probability density function (PDF), cumulative distribution function (CDF) and average bit error probability (ABEP) for system with channel state information (CSI) relay are derived. Some numerical results are presented to show behavior of performance gain for the proposed system. Analytical exact and lower bound expression for the outage probability (OP) of CSI assisted relay are obtained and required numerical results are compared.
\end{abstract}

K e y w o r d s: dual hop cooperative system, CSI relay, shadowed Ricen fading

\section{INTRODUCTION}

Recently, non-regenerative dual-hop cooperative links have gained great interest in wireless communication systems in order to achieve required high data rate coverage, as well as to mitigate wireless channel impairments $[1,2]$. When the annoyances over direct transmission path are evident, the third terminal between the source terminal and destination one, may be used. The scheme is known as a cooperative scheme with dual-hop transmission link which enables better communication between nodes [2-4]. Dual-hop transmission systems can be classified into two main categories: regenerative and non-regenerative systems. In regenerative systems the relaying node decodes the signal and then transmits the detected version to the next node, whereas in non-regenerative systems the relaying node just amplifies and forwards the incoming signal. Furthermore, relays of nonregenerative systems are classified in two subcategories. On one hand, there are channel state information (CSI) assisted relays, which use the instantaneous CSI from the previous hop to limit the output gain leading to a power control of the retransmitted signal. On the other hand, there are fixed-gain relays which introduce a fixed gain and a variable signal power at the output, but have lower complexity compared to the former subcategory [2-5]. CSI-based relays can be used in land mobile satellite (LMS) systems.

There are a number of research papers on dual hop relaying systems over fading channels. The end-to-end outage probability (OP) as well as the other performances of dual-hop wireless systems with non-regenerative relay operating over Rayleigh [3, 4], Nakagami- $m$ [5-7] and mixed Rayleigh and Ricean [8] fading channels were evaluated. The performance of dual-hop systems with fixed gain [9] and CSI gain [10] relays over generalized- $K$ fading channel was also addressed. All previously mentioned papers studied performances of relaying systems in the presence of various type of fading. We examine the outage probability and average bit error probability (ABEP) of CSI-based non- regenerative dual-hop cooperative system over shadowed Ricean fading channels. Exact and lower bound expressions for evaluating OP are derived in this paper. Presented analytical results are numerically compared. Using an upper bound for the end-to-end signal-to-noise ratio (SNR), novel closed-form expressions for the probability density function (PDF), the moments and the moment-generating function (MGF) of the endto-end SNR are also obtained.

As previously mentioned, we focus on dual-hop nonregenerative CSI relaying systems over shadowed Ricean fading channels. This type of channel model is often used in describing performances of terrestrial systems with line of sight (LOS) paths and LMS systems. The random fluctuations of the signal envelope in narrow-band LMS channels can be described by different statistical models depending on the channel condition. Some of proposed statistical models refer to multipath and shadow fading, namely, multipath, shadow and multiplicative shadow fading [11-16]. The shadow fading is often modeled as lognormal [11-15]. In [16] multipath fading, caused by the weak scatter components propagated via different nonLOS paths together with the nonblocked LOS component, is analyzed. The LOS component is observed as Nakagami- $m$ variable. So, the power of the LOS component is a gamma random variable, which is an alternative to the lognormal distribution and can result in simpler statistical model with the same performance for practical cases of interest. Also, a Rice model with Nakagamidistributed LOS amplitude [16] constitutes a versatile model which not only agrees very well with measured LMS channel data, but also offers significant analytical

\footnotetext{
* Faculty of Electronic Engineering, Aleksandra Medvedeva 14, Niš, Serbia, caleksandra@gmail.com, anastasovjelena@gmail.com, stefanpnc@yahoo.com, mihajlo.stefanovic@elfak.ni.ac.rs, dejan.milic@elfak.ni.ac.rs
} 


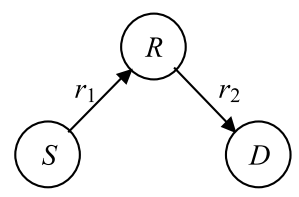

Fig. 1. Model of system

and numerical advantages for system performance predictions, design issues, etc. Therefore, dual hop relaying systems in proposed LMS fading environment are the main focus of the paper.

The remainder of this paper is organized as follows. The channel model is presented in Section 2, which also gives detailed discussion of nonregenerative dual-hop systems in Ricean fading and shadowing environment. In Section 3, new lower bound expressions CSI-assisted relaying communication systems are obtained for the probability density function, the cumulative distribution function $(\mathrm{CDF})$, the moments and the moment-generating function of bounded end-to-end SNR are derived. In this section we also derive the exact expression for the OP. In Section 4, we present and discuss numerical results for the outage probability of end-to-end SNR. Based on bounded statistics, numerical results for ABEP are also presented. Finally, our conclusions are drawn in Section 5.

\section{SYSTEM AND CHANNEL MODEL}

The model of wireless communication system where terminal $R$ relays signal from terminal $S$ to terminal $D$, considered in this paper, is shown in Fig. 1.

The source terminal $S$ communicates with the destination terminal $D$ over the cooperative path. Period of transmission is divided into two signaling intervals. During the first interval, terminal $S$ communicates with terminal $R$ and during the second interval, terminal $R$ communicates with the terminal $D[2]$. Assuming that $S$ transmits signal with an average power normalized to unity, the instantaneous equivalent SNR of dual-hop path can be expressed as [5]

$$
\gamma_{\mathrm{end}}=\frac{\left(r_{1}^{2} / N_{0,1}\right)\left(r_{2}^{2} / N_{0,2}\right)}{\left(r_{2}^{2} / N_{0,2}\right)+\left(1 / g^{2} N_{0,1}\right)},
$$

where $r_{i}$ is the fading amplitude of the $i$-th path, $i=$ 1,$2 ; N_{0, i}$ is the one-sided power spectral density of the additive white Gaussian noise at the input of $R$ and $D$, and $g$ is the gain of the relay. CSI-based relaying system aims to maintain a constant instantaneous output power for the retransmitted signal. In this case, the scaling gain is set to [5]

$$
g=\frac{1}{r_{1}^{2}+N_{0,1}},
$$

where the relay simply amplifies the received signal. As such, the instantaneous end-to-end SNR of the two hops can be rewritten as $[3,5]$

$$
\gamma_{\mathrm{end}}=\frac{\gamma_{1} \gamma_{2}}{\gamma_{1}+\gamma_{2}+1}
$$

Since $r_{i}$ is modeled as Ricean-shadowed variable, the instantaneous $\mathrm{SNR}, \gamma_{i}=r_{i}^{2} / N_{0, i}$, is a distributed random variable with PDF given by [16]

$$
\begin{aligned}
f_{\gamma_{i}}(\gamma)=\left(\frac{2 b_{i} m_{i}}{2 b_{i} m_{i}+\Omega_{i}}\right)^{m_{i}} \frac{1}{2 b_{i}} \exp \left(-\frac{\gamma}{2 b_{i}}\right) \times \\
{ }_{1} F_{1}\left(m_{i}, 1, \frac{\Omega_{i} \gamma}{2 b_{i}\left(2 b_{i} m_{i}+\Omega_{i}\right)}\right),
\end{aligned}
$$

where $2 b_{i}$ is the average power of scatter component per hop, $\Omega_{i}$ is the average power of LOS component per hop, $m_{i}$ is Nakagami fading parameter and ${ }_{1} F_{1}(\cdot, \cdot, \cdot)$ is the confluent hypergeometric function [17, 9.14/1)]. Traditionally, Nakagami model for multipath fading [5] $m$ changes over the limited range of $m \geq 0.5$, while this model allows $m$ to vary over the wider range of $m \geq$ 0 [16]. This enables evaluated PDF, (4), to accurately approximate a great variety of LOS conditions in LMS channels. For $0<m<\infty$ suburban and rural areas with partial obstruction of the LOS can be described. The extreme cases of $m=0$ or $m=\infty$ refer to model urban areas with complete obstruction or open areas with no obstruction of the LOS, respectively. Of course, these extreme cases cannot be met in practice. Moreover, CDF of the instantaneous SNR can be calculated as

$$
F_{\gamma_{i}}(\gamma)=\int_{0}^{\gamma} f_{\gamma_{i}}(x) \mathrm{d} x
$$

Using the infinite series representation of ${ }_{1} F_{1}(\cdot, \cdot, \cdot)$ (9.210/1)], CDF per hop can be derived

$$
\begin{aligned}
F_{\gamma_{i}}(\gamma) & =\left(\frac{2 b_{i} m_{i}}{2 b_{i} m_{i}+\Omega_{i}}\right)^{m_{i}} \times \\
& \sum_{k=0}^{\infty} \frac{\Gamma\left(m_{i}+k\right)}{(k !)^{2} \Gamma\left(m_{i}\right)}\left(\frac{\Omega_{i}}{2 b m_{i}+\Omega_{i}}\right)^{k} \Psi\left(1+k, \frac{\gamma}{2 b_{i}}\right)
\end{aligned}
$$

where $\Gamma(\cdot)$ is the Gamma function $[17,(8.31)]$ and $\Psi(\cdot, \cdot)$ the incomplete Gamma function [17, (8.350/1)]. Number of terms required for an accuracy at $6^{\text {th }}$ place of decimal digit in the numerical evaluation of (6) are presented in Table 1.

Table 1. Terms need to be summed in (6) to achieve accuracy at the $6^{\text {th }}$ significant digit

\begin{tabular}{cccc}
\hline$\gamma(\mathrm{dB})=$ & -10 & 0 & 10 \\
\hline Heavy shadowing & 2 & 3 & 3 \\
Overall shadowing & 3 & 5 & 9 \\
Average shadowing & 5 & 11 & 20 \\
Light shadowing & 5 & 11 & 19 \\
\hline
\end{tabular}




\section{PERFORMANCE ANALYSIS}

In noise-limited systems, outage probability is defined as the probability that the instantaneous end-to-end SNR, $\gamma_{\text {end }}$, falls below a predetermined protection ratio, $\gamma_{\text {th }}$. Consequently, OP is given by [5]

$$
\begin{aligned}
& P_{\text {exact }}=F\left(\gamma_{\mathrm{th}}\right)= \\
& \int_{0}^{\infty} F_{\gamma_{1}}\left(\frac{\gamma_{1} \gamma 2}{\gamma_{1}+\gamma_{2}+1} \leq \gamma_{\mathrm{th}} \mid \gamma_{2}\right) f_{\gamma_{2}}\left(\gamma_{2}\right) \mathrm{d} \gamma_{2}= \\
& \int_{0}^{\gamma_{\mathrm{th}}} F_{\gamma_{1}}\left(\gamma_{1} \geq \frac{\gamma_{\mathrm{th}}\left(\gamma_{2}+1\right)}{\gamma_{2}-\gamma_{\mathrm{th}}} \mid \gamma_{2}\right) f_{\gamma_{2}}\left(\gamma_{2}\right) \mathrm{d} \gamma_{2}+ \\
& \int_{\gamma_{\mathrm{th}}}^{\infty} F_{\gamma_{1}}\left(\gamma_{1} \leq \frac{\gamma_{\mathrm{th}}\left(\gamma_{2}+1\right)}{\gamma_{2}-\gamma_{\mathrm{th}}} \mid \gamma_{2}\right) f_{\gamma_{2}}\left(\gamma_{2}\right) \mathrm{d} \gamma_{2}= \\
& =I_{1}+I_{2} .
\end{aligned}
$$

It is obvious that the first integral in (7) is equal to $F_{\gamma_{2}}\left(\gamma_{\mathrm{th}}\right)$. The second integral can be solved representing ${ }_{1} F_{1}(\cdot, \cdot, \cdot)$ and $\Psi(\cdot, \cdot)$ through series expressions $[17$, $(9.210 / 1)$ and $(8.352 / 1)$, respectively], and then applying $[17,3.471 / 9]$

$$
\begin{gathered}
I_{2}=\left(\frac{2 b_{1} m_{1}}{2 b_{1} m_{1}+\Omega_{1}}\right)^{m_{1}}\left(\frac{2 b_{2} m_{2}}{2 b_{2} m_{2}+\Omega_{2}}\right)^{m_{2}} \times \\
\frac{1}{2 b_{2}} \sum_{k, l=0}^{\infty} \frac{\Gamma\left(m_{1}+k\right) \Gamma\left(m_{2}+l\right)}{\Gamma\left(m_{1}\right) \Gamma\left(m_{2}\right)(k !)^{2}(l !)^{2}}\left(\frac{\Omega_{1}}{2 b_{1} m_{1}+\Omega_{1}}\right)^{k} \times \\
\left(\frac{\Omega_{2}}{2 b_{2} m_{2}+\Omega_{2}}\right)^{l}\left[\Gamma\left(1+l, \frac{\gamma_{\mathrm{th}}}{2 b_{2}}\right) \Gamma(1+k)-\right. \\
\sum_{p=0}^{k} \frac{1}{p !}\left(\frac{1}{2 b_{2}}\right)^{l} k ! \exp \left(-\gamma_{\mathrm{th}}\left(\frac{1}{2 b_{1}}+\frac{1}{b_{2}}\right)\right) \times \sum_{i_{1}=0}^{l} \sum_{i_{2}=0}^{p}\left(\begin{array}{c}
l \\
i_{1}
\end{array}\right)\left(\begin{array}{c}
p \\
i_{2}
\end{array}\right) 2 \gamma_{\mathrm{th}}^{l-i_{1}}\left(\gamma_{\mathrm{th}}+1\right)^{i_{1}} \times \\
\left(\frac{b_{2} \gamma_{\mathrm{th}}\left(\gamma_{\mathrm{th}}+1\right)}{b_{1}}\right)^{\left(i_{1}-i_{2}+1\right) / 2} K_{i_{2}-i_{1}-1}\left(\sqrt{\left.\frac{\gamma_{\mathrm{th}}\left(\gamma_{\mathrm{th}}+1\right)}{b_{1} b_{2}}\right)}\right] .
\end{gathered}
$$

where $K_{i_{2}-i_{1}-1}(\cdot)$ is the $\left(i_{2}-i_{1}-1\right)^{\text {th }}$ order modified Bessel function of the second kind $[17,(8.407)]$. So, the CDF expression (7) is not easily tractable due to slow convergence for achieving required accuracy. Fortunately, this form can be approximated by its upperbound $\gamma_{b}$ as follows [8]

$$
\gamma_{\text {end }} \leq \gamma_{b}=\min \left(\gamma_{1}, \gamma_{2}\right)
$$

A physical interpretation of this bound is that at high SNR region, the hop with the weakest SNR determinates the end-to-end system performance. This approximation, is adopted in many recent papers $e g[7,8,10]$ and is shown to be accurate enough, specially at medium and high SNR values. The relay fade-shadowed channels are often independent, so the PDF of $\gamma_{b}$ is given by [18]

$$
\begin{aligned}
& f_{\text {bound }}\left(\gamma_{b}\right)=f_{\gamma_{1}}\left(\gamma_{b}\right)\left(1-F_{\gamma_{2}}\left(\gamma_{b}\right)\right)+ \\
& f_{\gamma_{2}}\left(\gamma_{b}\right)\left(1-F_{\gamma_{1}}\left(\gamma_{b}\right)\right)
\end{aligned}
$$

and CDF eg OP is given by $[10,18]$

$$
\begin{aligned}
P_{\text {bound }}=F\left(\gamma_{\mathrm{th}}\right)=F_{\gamma_{1}}\left(\gamma_{\mathrm{th}}\right)+ & F_{\gamma_{2}}\left(\gamma_{\mathrm{th}}\right)- \\
& F_{\gamma_{1}}\left(\gamma_{\mathrm{th}}\right) F_{\gamma_{2}}\left(\gamma_{\mathrm{th}}\right) .
\end{aligned}
$$

Also, starting from the basic definition, $m_{n}=\mathrm{E}\left(\gamma^{n}\right)$ [18], the $n$th order moment $m_{n}$ of $\gamma$ can be evaluated using

$$
m_{n}=\int_{0}^{\infty} \gamma^{n} f_{\text {bound }}(\gamma) \mathrm{d} \gamma
$$

Substituting (10) in (12), integrals in the forms $I_{1}=\int_{0}^{\infty} x^{\alpha} \exp \left(-A_{1} x\right)_{1} F_{1}\left(B_{1}, B_{2}, A_{2} x\right) \mathrm{d} x$ and $I_{2}=\int_{0}^{\infty} x^{\alpha} \exp \left(-A_{1} x\right)_{1} F_{1}\left(B_{1}, B_{2}, A_{2} x\right) \gamma\left(C_{1}, C_{2} x\right) \mathrm{d} x$ need to be solved. The integral $I_{1}$ can be solved representing $\exp (\cdot)$ and ${ }_{1} F_{1}(\cdot, \cdot, \cdot)$ by MeijerG functions $[19$, (01.03.26.0004.01) and (07.20.26.0005.01), respectively] and then applying [19, (07.34.21.0011.01)]. The integral $I_{2}$ can be solved using the infinite series representation of ${ }_{1} F_{1}(\cdot, \cdot, \cdot)[17,(9.210 / 1)]$ and representing $\Psi(\cdot, \cdot)$ and $\exp (\cdot)$ by MeijerG functions [19, (07.20.26.0005.01) and (01.03.26.0004.01, respectively]. Then we have applied $[19,(07.34 .21 .0011 .01)]$ and after some mathematical manipulations the $n$th order moment, $m_{n}$, is derived in this form

$$
\begin{aligned}
& m_{n}=\sum_{i=1}^{2} \frac{\pi\left(2 b_{i}\right)^{m_{i}+n} m_{i}^{m_{i}}}{\Gamma\left(m_{i}\right)\left(2 b_{i} m_{i}+\Omega_{i}\right)^{m_{i}-1} \Omega_{i}} \times \\
& G_{3,3}^{2,1}\left[\frac{2 b_{i} m_{i}+\Omega_{i}}{\Omega_{i}} \mid \begin{array}{ccc}
0, & 0, & -1 / 2 \\
n, & m_{i}-1, & -1 / 2
\end{array}\right]- \\
& \sum_{\substack{i, j=1 \\
i \neq j}}^{2}\left(\sum_{k, l=0}^{\infty} \frac{\left(2 b_{i}\right)^{n_{i}+n-1} m_{i}^{m_{i}}\left(2 b_{j}\right)^{m_{j}+1} m_{j}^{m_{j}}}{\left(2 b_{i} m_{i}+\Omega_{i}\right)^{m_{i}+l}\left(2 b_{j} m_{j}+\Omega_{j}\right)^{m_{j}+k}} \times\right. \\
& \left.\frac{\Gamma\left(m_{i}+l\right) \Gamma\left(m_{j}+k\right) \Omega_{j}^{k} \Omega_{i}^{l}}{\Gamma\left(m_{i}\right) \Gamma\left(m_{j}\right)(k !)^{2}(l !)^{2}} G_{2,2}^{2,1}\left[\frac{b_{j}}{b_{i}} \mid \begin{array}{rr}
-(1+k), & 0 \\
(n+l), & -1
\end{array}\right]\right) .
\end{aligned}
$$

Using (13), the amount of fading (AoF), generally known as a measure of the severity of fading, can be expressed in the form of infinite series as $A_{F}=m_{4} / m_{2}^{2}-1$. For calculating lower bound average bit-error or average symbol-error probability (ABEP or SEP) of several modulation schemes, we have derived MGF. Substituting (10) in $M_{\gamma}(g)=\int_{0}^{\infty} e^{-g \gamma} f_{\text {bound }}(\gamma) \mathrm{d} \gamma \geq 0$ and using the same transformations and mathematical manipulations as in solving (12), we get

$$
\begin{aligned}
& M_{\gamma}(g)=\sum_{i=1}^{2} \frac{\pi\left(2 b_{i} m_{i}\right)^{m_{i}}}{\Gamma\left(m_{i}\right)\left(2 b_{i} m_{i}+\Omega_{i}\right)^{m_{i}-1} \Omega} \times \\
& G_{3,3}^{2,1}\left[\frac{\left(2 b_{i} m_{i}+\Omega_{i}\right)\left(g 2 b_{i}+1\right)}{\Omega_{i}} \mid \begin{array}{ccc}
0, & 0, & -1 / 2 \\
n, & m_{i}-1, & -1 / 2
\end{array}\right]- \\
& \sum_{i, j=1, i \neq j}^{2}\left(\sum_{k, l=0}^{\infty} \frac{\left(2 b_{i}\right)^{m_{i}-l-1} m_{i}^{m_{i}}\left(2 b_{j}\right)^{m_{j}+l+1} m_{j}^{m_{j}}}{\left(2 b_{i} m_{i}+\Omega_{i}\right)^{m_{i}+l}\left(2 b_{j} m_{j}+\Omega_{j}\right)^{m_{j}+k}} \times\right. \\
& \frac{\Gamma\left(m_{i}+l\right) \Gamma\left(m_{j}+k\right) \Omega_{j}^{k} \Omega_{i}^{l}}{\Gamma\left(m_{i}\right) \Gamma\left(m_{j}\right)(k !)^{2}(l !)^{2}} G_{2,2}^{2,1} \times \\
& \left.\left[\frac{\left(g 2 b_{i}+1\right) b_{j}}{b_{i}} \mid \begin{array}{cc}
-(1+k+l), & -l \\
0, & -(1+l)
\end{array}\right]\right) \text {. }
\end{aligned}
$$




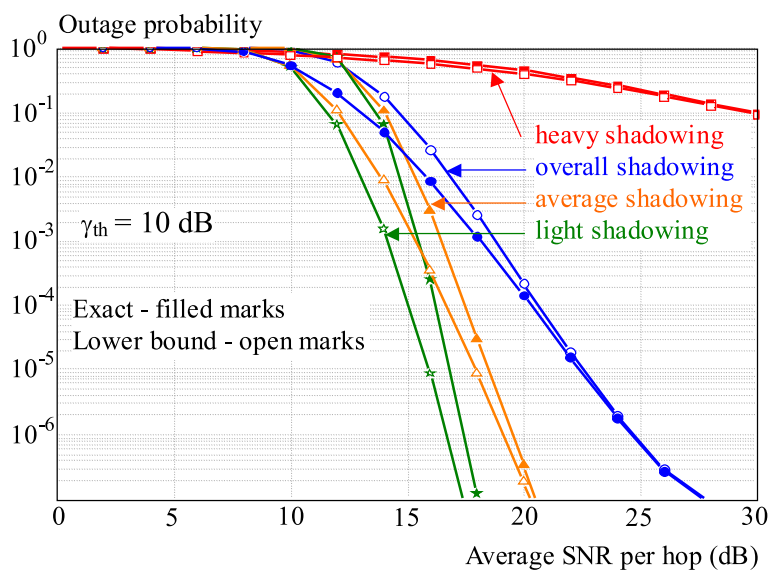

Fig. 2. Outage probability (exact and lower bound) for different shadowing conditions

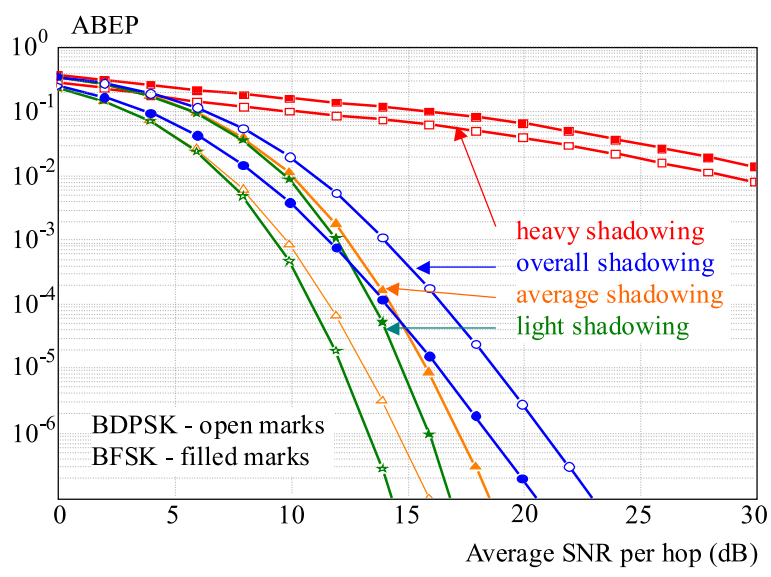

Fig. 4. ABEP bounds for BDPSK and BFSK for different shadowing conditions

\section{NUMERICAL RESULTS}

In this section, numerical results for the $\mathrm{OP}$ and the ABEP for binary modulation schemes are presented. We focus on four different shadowing scenarios, corresponding to Loo's model (refer to [16]), namely, infrequent light shadowing, frequent heavy shadowing, overall results and average shadowing scenario. The parameters we have used in evaluating numerical results are listed in Table III in [16]. This parameters were confirmed with Loo's empirical results and also have been used in several studies, for example in [10], for performance analysis of dual-hop system over generalized-K fading channels. So, this observation indicate the utility of our model.

In Fig. 2 the OP is plotted as a function of the average SNR per hop $\left(\bar{\gamma}_{1}=\bar{\gamma}_{2}=\bar{\gamma}\right)$, assuming $\gamma_{\text {th }}=10 \mathrm{~dB}$. As it can be observed, OP improves as the amount of shadowing decreases from heavy $\left(m_{1}=m_{2}=0.739, b_{1}=b_{2}=\right.$ $0.063)$ to average $\left(m_{1}=m_{2}=10.1, b_{1}=b_{2}=0.126\right)$ and then to overall $\left(m_{1}=m_{2}=5.21, b_{1}=b_{2}=0.278\right)$ and light $\left(m_{1}=m_{2}=19.4, b_{1}=b_{2}=0.158\right)$ shadowing condition. Moreover, curves for the exact and lower bounded outage performance are depicted for comparation purposes. It is obvious that the difference between exact value of the OP evaluated using (7) and lower bound

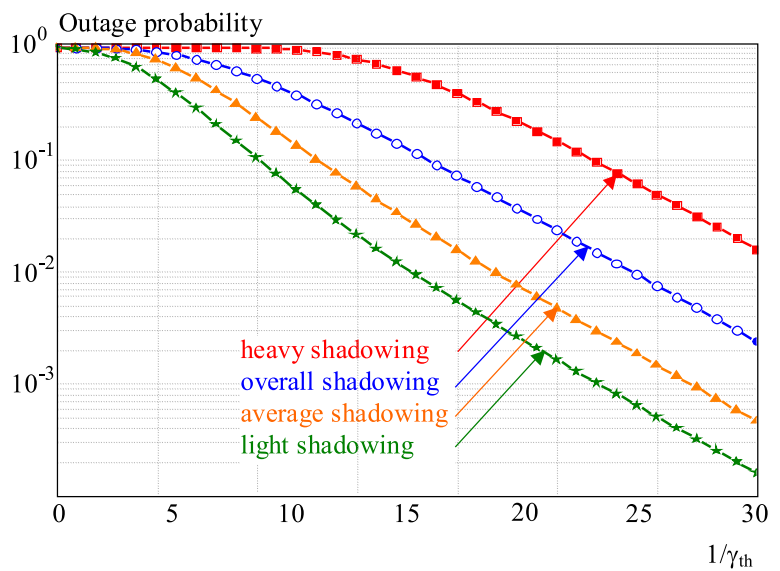

Fig. 3. Outage probability lower bound for different shadowing conditions

value evaluated using (11) becomes tighter with the increase of $\bar{\gamma}$. That differences get almost invisible at low values of $\bar{\gamma}$. Also, it can be observed that the outage bound performance gets tighter to exact outage as shadowing increases. From the Fig. 2, we clearly see an acceptable match between exact and lower bound curves. The analytical evaluation of bound OP (11) is significantly faster than the evaluation of exact OP (7), so our forward numerical results are based on bound evaluations.

In Fig. 3 lower bounds for the outage probability are plotted as a function of $1 / \gamma_{\mathrm{th}}$. We obtained this numerical results using lower bound expression (11). The parameters, we have used in evaluating, are: for heavy shadowing condition $m_{1}=m_{2}=0.739, b_{1}=b_{2}=0.063$, $\Omega=8.97 \times 10^{-4}$; for average $m_{1}=m_{2}=10.1, b_{1}=$ $b_{2}=0.126, \Omega=0.835$; for overall $m_{1}=m_{2}=5.21$, $b_{1}=b_{2}=0.278, \Omega=0.278$ and for light shadowing condition $m_{1}=m_{2}=19.4, b_{1}=b_{2}=0.158, \Omega=1.29$. As seen, the offered gain becomes marginal for heavy shadowing condition.

In Fig. 4 the ABEP of binary differential phase-shift keying (BDPSK), using $\bar{P}_{e}=0.5 M_{\gamma}(1)$, and noncoherent binary frequency-shift keying (BFSK), using $\bar{P}_{e}=$ $0.5 M_{\gamma}(0.5)$, is plotted as a function of $\bar{\gamma}$. It is evident that ABEP decreases, ie performance gain increases, as the amount of shadowing decreases. Also, Fig. 4 shows better performances of BDPSK modulation scheme compared to BFSK scheme.

\section{CONCLUSION}

In this paper, bounded performances for dual-hop transmission with CSI assisted relays operating over Ricean shadowed fading channels have been investigated. Novel lower bound expressions for the PDF, CDF and MGF for end-to-end bounded SNR have been derived. The series representation for evaluating exact outage probability of proposed system has been also obtained. Some numerical results are presented in order to improve analytical analysis. 


\section{REFERENCES}

[1] LANEMAN, J. N.-TSE, D. N. C.-WORNELL, G. W. : Cooperative Diversity in Wireless Networks Efficient Protocols and Outage Behavior, IEEE Trans. Inf. Theory 50(12) (2004), 3062-3080.

[2] HASNA, M. O.-ALOUINI, M.-S.: A Performance Study of Dual-Hop Transmissions with Fixed Gain Relays, IEEE Trans. Wireless Commun. 3(6) (2004), 1963-1968.

[3] HASNA, M. O.-ALOUINI, M.-S : End-to-End Performance of Transmission Systems with Relays over Rayleigh Fading Channels, IEEE Trans. Wireless Commun 2(6) (2003), 1126-1131.

[4] HASNA, M. O.-ALOUINI, M.-S : Harmonic Mean and End-toEnd Performance of Transmission Systems with Relays, IEEE Trans. Commun. 52(1) (2004), 130-135.

[5] TSIFtSis, T. A.-KARAGIANNIDIS, G. K.-MATHIOPOULOS, P. T.-KOTSOPOULOS, S. A: Nonregenerative DualHop Cooperative Links with Selection Diversity, EURASIP J. Wireless Commun. Network (2006), 1-8.

[6] HASNA, M. O.-ALOUINI, M.-S : Outage Probability of Multihop Transmission over Nakagami Fading Channels, IEEE Commun. Lett. 7(5) (2003), 216-218.

[7] KARAGIANNIDIS, G. K.-TSIFTSIS, T.-MALLIK, R. : Bounds for Multihop Relayed Communications in Nakagami-m Fading, IEEE Trans. Commun. 54(15) (2006), 18-22.

[8] SURAWEERA, H. A.-LOUIE, R. H. Y.-LI, Y.-KARAGIANNIDIS, G. K.-VUCETIC, B.: Two Hop Amplify and Forward Transmission in Mixed Rayleigh and Rician Fading Channels, IEEE Commun. Lett. 13(4) (2009), 227-229.

[9] KARAGIANNIDIS, G. K.: Performance bounds of Multihop Wireless Communications with Blind Relays over Generalized Fading Channels, IEEE Trans. Wireless Commun. 5(3) (2006), 498-503.

[10] PEPPAS, K. P.-DATSIKAS, C. K.-NISTAZAKIS, H. E.TOMBRAS, G.: Dual-Hop Relaying Communications over Generalized K Fading Channels, Journal of the Franklin Institute 347 (2010), 1643-1653.

[11] LOO, C.: A Statistical Model for a Land Mobile Satellite Link, IEEE Trans. Vehic. Technol 34 (1985), 122-127.

[12] CORAZZA, G. E.-VATAlaro, F.: A Statistical Model for Land Mobile Satellite Channels and its Application to Nongeostationary Orbit Systems, IEEE Trans. Vehic. Technol. 43 (1994), 738-742.

[13] VATALARO, F.: Generalized Rice-Lognormal Channel Model for Wireless Communications, Electron. Lett. 31 (1995), 1899-1900.

[14] LUTZ, E.-CYGAN, D.-DIPPOLD, M.-DOLAINSKY, F.PAPKE, W. : The Land Mobile Satellite Communication Channel - Recording, Statistics, and Channel Model, IEEE Trans. Vehic. Technol. 40 (1991), 375-386.

[15] BARTSR. M. AndSTUtzMAnW. L: Modeling and Simulation of Mobile Satellite Propagation, IEEE Trans. Antennas Propagat. 40 (1992), 375-382.

[16] ABDI, A.-LAU, W. C.-ALOUINI, M.-S.-KAVEH, M.: A New Simple Model for Land Mobile Satellite Channels: Firstand Second-Order Statistics, IEEE Trans. Wireless Commun. 2(2) (2003), 519-528.

[17] GRADSHTEYN, I.-RYZHIK, I. M. : Tables of Integrals Series and Products, sixth ed., Academic Press, New York, 2000.

[18] PAPOULIS, A.: Probability, Random Variables, and Stochastic Processes, $3^{\text {rd }}$ ed., (McGraw-Hill, New York, 1991.
[19] The Wolfram Functions Site, 2008. (Online) Available: http://functions.wolfram.com.

Received 31 December 2011

Aleksandra M. Cvetković was born in Niš, Serbia. She received BS and MS degrees in electrical engineering from Faculty of Electronic Engineering, Niš, Serbia, in 2001 and 2007, respectively. She is currently working at the Faculty of Electronic Engineering, Nis, Serbia, as an Assistant. Her research interests include wireless communication theory, statistical characterization and modeling of fading channels, performance analysis of diversity combining techniques, outage analysis of multi user wireless systems subject to interference. She has published several papers on the above subjects.

Jelena Anastasov was born in Vranje, Serbia in 1982. She received MSc degree in Electrical Engineering from the Faculty of Electronic Engineering, Niš, Serbia, in 2006 as the best graduated student of the generation 2001/2002. She is currently on PhD studies, Telecommunications Department, as Research Assistant at the Faculty of Electronic Engineering, $\mathrm{Niš}$, Serbia. Her research interests include performance analysis of wireless communication systems, analysis and modeling of fading channels and statistical signal processing. She has published several papers on the above subjects.

Stefan Panić was born in Pirot, Serbia, in 1983. He received MSc degree in electrical engineering from Faculty of Electronic Engineering, Niš, Serbia, in 2007. He joined the Department of Telecommunication, Faculty of Electronic Engineering, Niš, Serbia in 2008 as Research Assistant on joint project between Faculty of Electronic Engineering and Ministry of Science Republic of Serbia. His research interests are in mobile and multichannel communications, fading channels, and statistical signal processing. He has published several papers on the above subjects.

Mihajlo Stefanović was born in Niš, Serbia in 1947. He received $\mathrm{BSc}, \mathrm{MSc}$ and $\mathrm{PhD}$ degrees in electrical engineering from the Faculty of Electronic Engineering, Department of Telecommunications, University of Niš, Serbia, in 1971, 1976 and 1979, respectively. His primary research interests are statistical communication theory, optical and satellite communications. His areas of interest also include applied probability theory, optimal receiver design and synchronization. He has written or co-authored a great number of journal publications. Dr Stefanovic is a Professor at the Faculty of Electronic Engineering, Department of Telecommunications, University of Niš, Serbia.

Dejan Milić was born in Niš, Serbia in 1972. He received $\mathrm{BS}, \mathrm{MS}$, and PhD degrees in electrical engineering from the Faculty of Electronic Engineering, University of Niš, Serbia, in 1997, 2001 and 2005, respectively. He is currently an Assistant Professor at the Department of Telecommunications at the same Faculty, and there he teaches optical communications and coherent communication systems. Other areas of research include communication theory, modulation and wireless communications. 